\title{
Mesenteric Venous Thrombosis After Laparoscopic Robotic-Assisted Colectomy
}

\author{
Jonathan Giannone, MD, Rami Lutfi, MD, FACS \\ Department of Surgery, Mercy Hospital and Medical Center, Chicago, IL, USA (all authors).
}

\begin{abstract}
Background: Mesenteric venous thrombosis has been a reported rare postoperative complication after laparoscopic surgery and may lead to increased patient morbidity and possible mortality.

Methods: We report a case highlighting the postoperative presentation of mesenteric venous thrombosis after laparoscopic robotic-assisted colectomy and its management.

Results: We present a case of a 52-year-old woman who underwent robotic-assisted right colectomy after screening colonoscopy found an adenocarcinoma in the cecum. She was discharged on postoperative day 4 and returned to the emergency department on postoperative day 20 with diffuse abdominal pain and nausea/vomiting. A computed tomography scan showed thrombosis and complete occlusion of her superior mesenteric vein extending to the portal vein.
\end{abstract}

Conclusion: Mesenteric venous thrombosis is a rare but highly morbid postoperative complication after laparoscopic surgery. It should be considered in the differential diagnosis of delayed postoperative presentation with abdominal pain.

Key Words: Mesenteric venous thrombosis, Laparoscopic surgery, Colectomy, Robotic surgery.

Citation Giannone J, Lutfi R. Mesenteric venous thrombosis after laparoscopic robotic-assisted colectomy. CRSLS e2014.00097. DOI: 10.4293/CRSLS.2014.00097.

Copyright (C) 2014 SLS This is an open-access article distributed under the terms of the Creative Commons Attribution-Noncommercial-ShareAlike 3.0 Unported license, which permits unrestricted noncommercial use, distribution, and reproduction in any medium, provided the original author and source are credited.

Address correspondence to: Jonathan Giannone, MD, Department of Surgery, Mercy Hospital and Medical Center, Chicago, IL 60616. Telephone: 847-712-5515, E-mail: atuneman@hotmail.com

\section{INTRODUCTION}

A continued growing number of colectomies are now being performed laparoscopically, and some of these are done with the assistance of robotics. A rare postoperative complication that has been reported, especially after laparoscopic surgery, is mesenteric venous thrombosis (MVT). This is a potentially fatal occurrence when there are delays in diagnosis. Previous reports in the literature describe MVT as most commonly seen after laparoscopic splenectomy with direct manipulation of the mesenteric venous system. ${ }^{1}$ There have been 3 reported laparoscopic colectomies in the literature with postoperative MVT. ${ }^{2-4}$ We present the first reported robotic-assisted colectomy with this rare complication.

\section{CASE REPORT}

A 52-year-old woman was found to have a cecal adenocarcinoma on a screening colonoscopy. She was morbidly obese but otherwise healthy, and a 20-pack-year smoker who quit 1 month before surgery. She underwent uncomplicated robotic-assisted right colectomy, with an on console time of 57 minutes and an average pneumoperitoneum of $15 \mathrm{~mm} \mathrm{Hg}$. Her cancer was T4NOM0 (14 negative lymph nodes). She had an uncomplicated hospital course. On postoperative day 1, the patient was started on $5000 \mathrm{U}$ of subcutaneous heparin every 8 hours. She continued to wear Sequential Compression Devicess and was ambulating early. On postoperative day 4 she was discharged home and then returned to the emergency department on postoperative day 20 with acute, sharp, diffuse abdominal pain and nausea and vomiting for 1 day. She reported recent loose stools and no fevers. Her physical examination was significant for a soft, obese, nondistended, but diffusely tender, abdomen with no peritoneal signs. Admission laboratory findings were all within normal limits. A computed tomography scan of the abdomen and pelvis demonstrated thrombus within the superior mesenteric vein (SMV) with the typical "target sign" extending to the proximal confluence of the portal vein (Figures 1, 2 and 


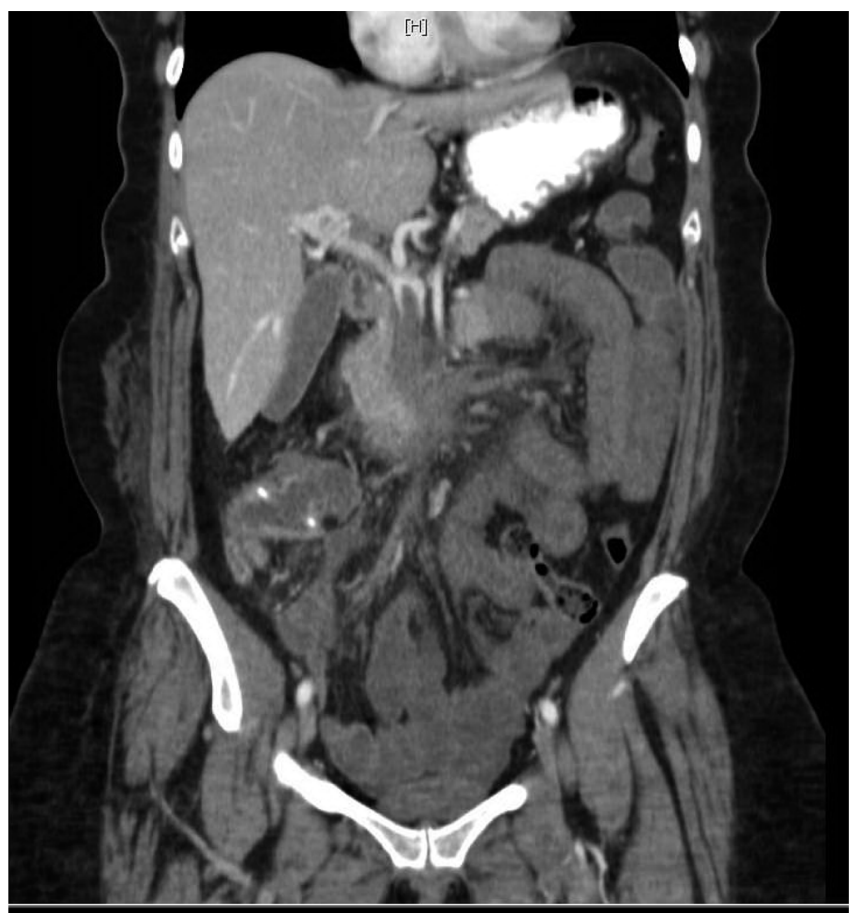

Figure 1. Thrombosis of SMV to the confluence of the portal vein and ischemic enteritis of the small bowel.

3). There was also thickening of the small bowel without evidence of transmural ischemia. She was admitted to the intensive care unit and began receiving intravenous heparin. She was maintained as "nothing by mouth", had a nasogastric tube placed, and started on total parenteral nutrition. She had slow but gradual improvement in her clinical condition and was advanced to oral intake on hospital day 6. Her hypercoaguable workup results were negative. She was transitioned to fondaparinux injections for long-term anticoagulation therapy and was discharged home on hospital day 13 after complete return of normal bowel function. She was seen last $\sim 1$ month after discharge and was found to be doing well.

\section{DISCUSSION}

MVT is a major cause of acute mesenteric ischemia, accounting for $5 \%$ to $15 \%$ of cases. ${ }^{5}$ It is a rare but potentially fatal complication after laparoscopic surgery and may require reoperation and resection of dead bowel. A recent literature review by James et al showed MVT to occur after 7 Roux- en-Y gastric bypasses, 5 Nissen fundoplication procedures, 3 colectomies, 2 cholecystectomies, and 1 appendectomy. 6 The mean age of patients was 42 years, and the average day of presentation was 14 days postoperatively. The main presenting symptom was diffuse ab-

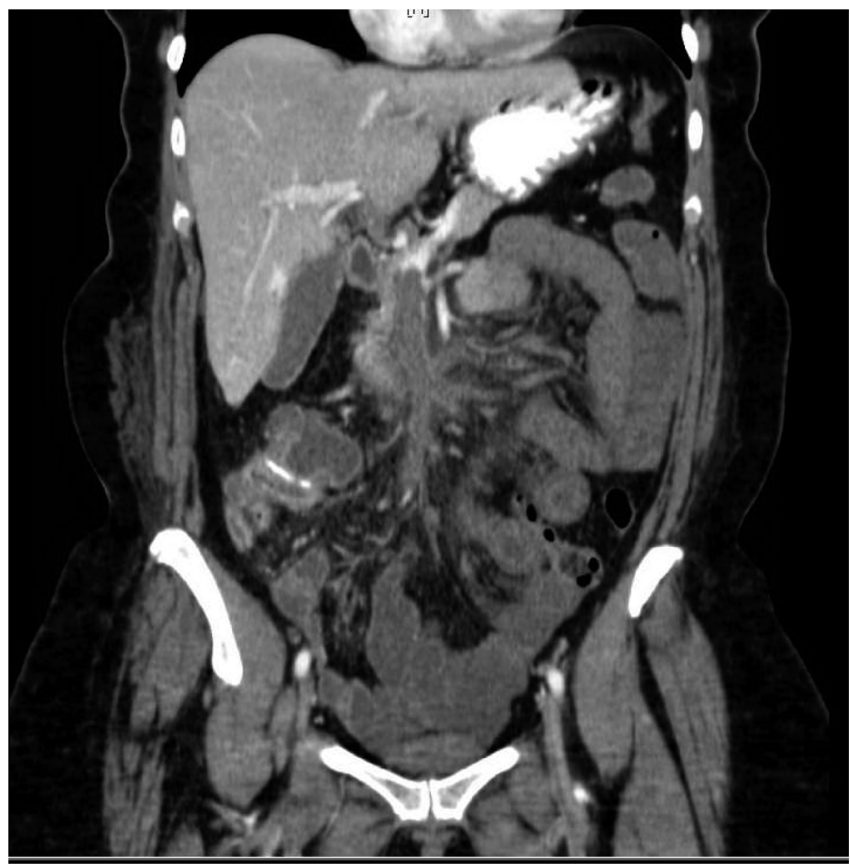

Figure 2. Complete superior MVT.

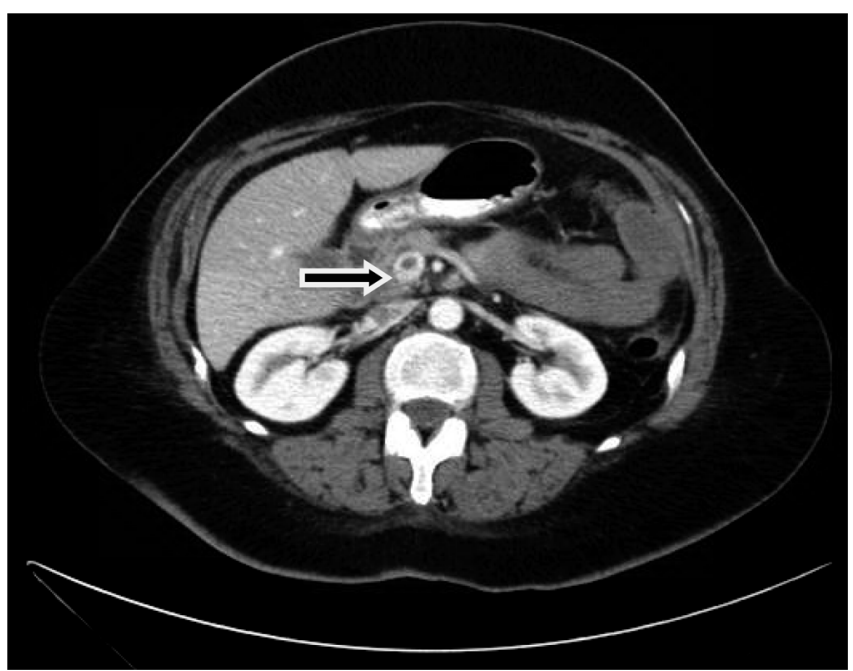

Figure 3. SMV thrombosis with typical "target sign." The lack of bowel wall enhancement is consistent with ischemic enteritis.

dominal pain; however, nausea, vomiting, and diarrhea were also commonly seen. Predisposition toward venous thrombosis was seen in 11 of these cases. Laboratory studies were not found to be helpful, although findings of mild leukocytosis and elevated liver enzymes were typical.

There have been 3 previously reported cases of MVT after laparoscopic colectomy (2 for precancerous polyp ${ }^{2,3}$ and 1 for diverticulitis ${ }^{4}$. Two of these patients were found to 
have prothrombotic states, unlike our patient who had negative workup results.

It has been hypothesized that the increased inflammatory state of the patient, as seen in the case of diverticulitis, may be a risk factor for postoperative MVT. ${ }^{4}$ However, this has only been seen in one of the now 4 reported cases of postoperative MVT after laparoscopic colectomy, making it a less likely cause. Our surgical case, unlike the other reported cases, was performed for a known adenocarcinoma, which by itself could cause an oncologic hypercoaguable state. Given this thrombophilia, following appropriate tumor markers both pre- and postoperatively may be another method of assessing a patient's risk for thrombosis.

There have been several postulations about why laparoscopic surgery increases the risk of MVT. Pneumoperitoneum with pressures up to $14 \mathrm{~mm} \mathrm{Hg}$ have been shown to decrease portal blood flow by $>50 \% .^{7}$ In our experience, laparoscopic colectomies are performed with insufflation pressures ranging from 12 to $15 \mathrm{~mm} \mathrm{Hg}$ and would not be technically feasible with pressures lower than those. Hypercapnia secondary to peritoneal insufflation with carbon dioxide has been shown to increase portal pressures, causing mesenteric vasoconstriction. ${ }^{8}$ Undiagnosed prothrombotic states have been discovered in patients with postoperative MVT. A recent literature review demonstrates that $44 \%$ of reported cases involved patients with unkown hypercoaguable disorders. ${ }^{9}$ Our patient did not have a prothombotic state but was morbidly obese and a former smoker. These two risk factors have been shown to be directly related to venous thrombosis. ${ }^{10,11}$ The length of the procedure and patient positioning have also been found to be potential risk factors for thrombosis in laparoscopic surgery.

The incidence of venous thrombosis after laparoscopic surgery varies according to the complexity of the procedure, as well as according to patient risk factors. ${ }^{12}$ There has been a recent investigation into discharging high-risk patients home with a course of pharmacologic venous thrombosis prophylaxis. Davenport et $\mathrm{al}^{13}$ reported that almost one-third of postoperative venous thrombotic events in colorectal patients occurred after discharge. Our patient may have benefitted from continued prophylaxis given her postoperative presentation and risk factors for thrombosis.

Diagnosis after clinical suspicion for postoperative MVT is confirmed with computed tomography scan. If the patient is clinically stable without clinical signs of peritonitis, a conservative, nonoperative management approach should be fol- lowed. The patient is observed in an intensive care unit with bowel rest and started early on anticoagulation therapy. In cases of a failed response to systemic anticoagulation, transjugular intrahepatic thrombolytic therapy has been described with success. ${ }^{3}$ Immediate use of thrombolytics on diagnosing MVT has been described with success in patients requiring laparotomy for peritoneal signs and having operatively placed mesenteric venous catheters for thrombolysis. ${ }^{14,15}$ This use of early thrombolytics resulted in limited bowel resections. Our patient was treated nonoperatively and had a return to bowel function on hospital day 6. Parenteral nutrition should be started early in all cases. Her relatively benign course with this postoperative complication can be attributed to a prompt diagnosis and treatment with appropriate supportive care. Anticoagulation was continued long term and may be for the rest of the patient's life based on her risk factors.

\section{CONCLUSION}

MVT after laparoscopy is a rare, potentially lethal complication. The laparoscopic surgeon should be aware of common presenting clinical factors because early diagnosis and management are critical to avoid the associated high morbidity and mortality rates.

Dr. Lutfi is a consultant for Ethicon, Allergan, and Gore.

\section{References:}

1. Ikeda M, Sekimoto M, Takiguchi S, et al. High incidence of thrombosis of the portal venous system after laparoscopic splenectomy. a prospective study with contrast enhanced CT scan. Ann Surg. 2005;241(2):217-218.

2. Millikan KW, Szczerba SM, Dominiguez JM, McKenna R, Roriq JC. Superior mesenteric and portal vein thrombosis following laparoscopic assisted right hemicolectomy. Dis Colon Rectum. 1996;39:1171-1175.

3. Poultsides GA, Lewis WC, Feld R, Walters DL, Cherry DA, Ruby ST. Portal vein thrombosis after laparoscopic colectomy: thrombolytic therapy via the superior mesenteric vein. Am Surg. 2005;71(10):856-860.

4. Baixauli J, Delaney CP, Senagore AJ, Remzi FH, Fazio VW. Portal vein thrombosis after laparoscopic sigmoid colectomy for diverticulitis: report of a case. Dis Colon Rectum. 2003;46:550-553.

5. Kairaluoma MI, Karkola P, Heikkinen D, Huttunen R, Mokka RE, Larmi TK. Mesenteric infarction. Am J Surg. 1977;133:188-193.

6. James AW, Rabl C, Westphalen AC, Fogarty PF, Posselt AM, Campos GM. Portomesenteric venous thrombosis after laparoscopic surgery: a systematic literature review. Arch Surg. 2009; 144(6):520-526. 
7. Takagi S. Hepatic and portal vein blood flow during carbon dioxide pneumoperitoneum for laparoscopic hepatectomy. Surg Endosc. 1998;12:427-431.

8. Johnson CM, de la Torre RA, Scott JS, Johansen T. Mesenteric venous thrombosis after laparoscopic Roux-en-Y gastric bypass. Surgery Obes Relat Dis. 2005;1:580-583.

9. Sucandy I, Gabrielsen JD, Petrick AT. Postoperative mesenteric venous thrombosis: potential complication related to minimal access surgery in a patient with undiagnosed hypercoagulability. North Am J Med Sci. 2010;2:329-332.

10. Enga KF, Braekkan SK, Hansen-Krone IJ, le Cessie S, Rosendaal FR, Hansen JB. Cigarette smoking and the risk of venous thromboembolism: the Tromso study. J Thromb Haemost. 2012;10:2068-2074.

11. Allman-Farinelli MA. Obesity and venous thrombosis: a review. Semin Thromb Hemost. 2011;37(8):903-907.
12. Nguyen NT, Owings JT, Gosselin R, et al. Systemic coagulation and fibrinolysis after laparoscopic and open gastric bypass. Arch Surg. 2001;136(8):909-916.

13. Davenport DL, Vargas HD, Kasten MW, Xenos ES. Timing and perioperative risk factors for in-hospital and post-discharge venous thromboembolism after colorectal cancer resection. Clin Appl Thromb Hemost. 2012;18(6):569-575.

14. Kaplan JL, Weintraub SL, Hunt JP, Gonzalez A, Lopera J, Brazzini A. Treatment of superior mesenteric and portal vein thrombosis with direct thrombolytic infusion via an operatively placed mesenteric catheter. Am Surg. 2004;70:600-604.

15. Ozdogan M, Gurer A, Gokakin AK, Kulacoglu H, Aydin R. Thrombolysis via an operatively placed mesenteric catheter for portal and superior mesenteric vein thrombosis: report of a case. Surg Today. 2006;36(9):846-848. 